\title{
Low uptake of fluorodeoxyglucose in positron emission tomography/computed tomography in ovarian clear cell carcinoma may reflect glutaminolysis of its cancer stem cell-like properties
}

\author{
MASAKAZU SATO $^{1}$, KEI KAWANA ${ }^{1,2}$, KATSUYUKI ADACHI ${ }^{1}$, ASAHA FUJIMOTO ${ }^{1}$, \\ AYUMI TAGUCHI $^{1}$, TOMONA FUJIKAWA ${ }^{1}$, MITSUYO YOSHIDA ${ }^{1}$, HIROE NAKAMURA ${ }^{1}$, \\ HARUKA NISHIDA $^{1}$, TOMOKO INOUE ${ }^{1}$, JURI OGISHIMA ${ }^{1}$, SATOKO EGUCHI $^{1}$, AKI YAMASHITA ${ }^{1}$, \\ KENSUKE TOMIO $^{1}$, TAKAHIDE ARIMOTO ${ }^{1}$, OSAMU WADA-HIRAIKE ${ }^{1}$, KATSUTOSHI ODA ${ }^{1}$, \\ TAKESHI NAGAMATSU ${ }^{1}$, YUTAKA OSUGA ${ }^{1}$ and TOMOYUKI FUJII ${ }^{1}$ \\ ${ }^{1}$ Department of Obstetrics and Gynecology, Graduate School of Medicine, The University of \\ Tokyo, Bunkyo-ku, Tokyo 113-0033; ${ }^{2}$ Department of Obstetrics and Gynecology, \\ Nihon University School of Medicine, Itabashi-ku, Tokyo 173-8610, Japan
}

Received August 28, 2016; Accepted January 16, 2017

DOI: $10.3892 /$ or.2017.5398

\begin{abstract}
The characteristics of ovarian cancers that showed low activation of glycolysis were investigated. Using medical records of patients with ovarian cancers who had undergone fluorodeoxyglucose positron emission tomography/computed tomography (FDG-PET/CT) prior to their primary surgery at the University of Tokyo Hospital between 2010 and 2015, we identified cases with a low uptake of FDG in PET/CT. We considered the maximum standardized uptake value $\left(\mathrm{SUV}_{\max }\right)$ as the degree of glucose uptake. We investigated the properties which may account for the low activation of glycolysis in vitro. The expression level of alanine, serine, cysteine-preferring transporter 2 (ASCT2, a glutamine influx transporter), system L-type amino acid transporter 1 (LAT1, a glutamine efflux transporter) and glucose transporter 1 (GLUT1, a glucose influx transporter) were investigated by western blotting. The phosphorylation level of AMP-activated protein kinase (AMPK), which is one of the metabolic sensors, was also investigated. Most of the cases with a low uptake $\mathrm{SUV}_{\max }$ were limited to patients with ovarian clear cell carcinoma (CCC). We obtained cancer stem cell (CSC)-like properties from CCC cell lines, and compared the expression levels of transporters between non-CSCs and CSCs. Whereas the expression level of ASCT2
\end{abstract}

Correspondence to: Dr Kei Kawana, Department of Obstetrics and Gynecology, Nihon University School of Medicine, 30-1 Oyaguchi-Kamicho, Itabashi-ku, Tokyo 173-8610, Japan E-mail: kkawana-tky@umin.org

Key words: fluorodeoxyglucose positron emission tomography/computed tomography, ovarian cancer, clear cell carcinoma, glutaminolysis, cancer stem cells was nearly unchanged between non-CSCs and CSCs, the expression levels of LAT1 and GLUT1 were decreased in CSCs compared to non-CSCs. The phosphorylation level of AMPK was reduced in CSCs compared to non-CSCs. In conclusion, we suggested that ovarian CCC showed low activation of glycolysis, and this may reflect glutaminolysis of its CSC-like properties.

\section{Introduction}

The Warburg effect is a well-documented metabolic hallmark of cancer cells (1). Cancer cells are thought to exclusively activate glycolysis, even in the presence of adequate oxygen. One technique in clinical practice that takes advantage of glycolysis is fluorodeoxyglucose positron emission tomography/computed tomography (FDG-PET/CT) $(2,3)$. Normally, in cancer cells, glucose is transported into the cytoplasm via glucose transporters (GLUTs), especially GLUT1 (4). Unlike glucose, FDG is a glucose-like substance that is also transported into tissues but is not further metabolized, resulting in its accumulation in tissues with active glucose metabolism (5). By detecting the accumulation of FDG, FDG-PET/CT is used as a diagnostic aid to distinguish cancer cells from normal cells. One of the quantitative values that reflect the degree of FDG accumulation is the maximum standardized uptake value $\left(\mathrm{SUV}_{\text {max }}\right)$. Indeed, recent studies have suggested its diagnostic value. In addition, some studies have demonstrated a correlation between $\mathrm{SUV}_{\max }$ and patient prognosis (6-8).

Moreover, it is also well-known that cancer cells not only uptake the most glucose (glycolysis), but they also uptake the most glutamine (glutaminolysis) (9), which is the most abundant amino acid in serum, indicating that cancer cells do not always show activation of glycolysis (or a high $\mathrm{SUV}_{\max }$ ). Then we became interested in the characteristics of cancer cells with a low activation of glycolysis (low $\mathrm{SUV}_{\max }$ ). 
First, using medical records, we identified cases with a low $\mathrm{SUV}_{\max }$ among patients with ovarian cancer. We found that most of the cases were limited to patients with ovarian clear cell carcinoma (CCC). Indeed, some studies previously indicated that ovarian $\mathrm{CCC}$ had a lower $\mathrm{SUV}_{\max }$ or lower uptake of FDG compared to adenocarcinoma of other histological types $(5,10)$. Then we became interested in the biology of CCC which causes low activation of glycolysis, and we speculated that CCC had the properties which were likely to utilize glutaminolysis compared to glycolysis. To investigate this hypothesis, we conducted an in vitro experiment to clarify the biology, which could explain the low $\mathrm{SUV}_{\max }$ of CCC.

We obtained cells with cancer stem cell (CSC)-like properties by forming spheroids as previously described (11). We then used western blotting to compare the expression levels of transporters, which correlate with cancer metabolism. Whereas the expression level of alanine, serine, cysteine-preferring transporter 2 (ASCT2, a glutamine influx transporter) was nearly unchanged between non-CSCs and CSCs, the expression levels of system L-type amino acid transporter 1 (LAT1, a glutamine efflux transporter) and GLUT1, a glucose influx transporter were decreased in CSCs compared to non-CSCs. These results indicated that glutamine metabolism was essential in cells with CSC-like properties and that the low $\mathrm{SUV}_{\max }$ of CCC may reflect the metabolism of glutamine in CSCs.

In the present study, we used FDG-PET analysis and showed that ovarian CCC may rely on glutaminolysis, and we confirmed the significance of glutamine metabolism in ovarian $\mathrm{CCC}$ by an in vitro experiment. We suggest that ovarian $\mathrm{CCC}$ has a low uptake of FDG in FDG-PET/CT, and this may reflect glutaminolysis of its CSC-like properties.

\section{Materials and methods}

Patients. This study was approved by the Institutional Ethics Committee. All procedures involving human participants were in accordance with the Ethical Standards of the Institutional and/or National Research Committee and with the 1964 Helsinki Declaration and its later amendments or comparable ethical standards.

Medical records and data from the gynecological oncology database were retrospectively reviewed. Only epithelial tumors, which were confirmed by pathological examinations after surgery, were included.

First, patients with ovarian tumors who had undergone FDG-PET within one month prior to their primary surgery at the University of Tokyo Hospital between 2010 and 2012 were enrolled (13 cases of benign tumors, 11 cases of borderline tumors and 26 cases of malignant tumors). The $\mathrm{SUV}_{\max }$ of non-malignant tumors (including benign and borderline tumors) and malignant tumors were compared. A cut-off value was chosen based on a receiver operating characteristic (ROC) curve.

Second, patients who had undergone primary surgery at the University of Tokyo Hospital between 2012 and 2015 and who were thought to have a low $\mathrm{SUV}_{\max }$ were also enrolled. The definition of low or high $\mathrm{SUV}_{\max }$ is discussed in the Results.

FDG-PET/CT. Patients underwent PET/CT not only at the University of Tokyo Hospital but also at other imaging centers.
A representative protocol is as follows. Patients fasted for at least $4 \mathrm{~h}$ before being intravenously injected with $185-370 \mathrm{MBq}$ of FDG, and then rested for 50 min before evaluation. Patients were scanned when their plasma glucose levels were below $200 \mathrm{mg} / \mathrm{dl}$. Patients were assessed using an integrated Discovery ST Elite Performance PET/CT (GE Healthcare Japan Corp., Tokyo, Japan) scanner. Unenhanced CT images of 3.75-mm thick sections that matched the PET images were acquired from the head to the pelvic floor of each patient using a standard protocol. The PET images were reconstructed using 3D-OSEM VUE point HD (iteration no. 2, subset no. 21), with a $6 \mathrm{~mm}$ Full-Width Half-Maximum (FWHM) Gaussian in-plane post filter (GE Healthcare).

For semi-quantitative analysis, a region of interest was drawn for each lesion in the transverse section where the lesion seemed to have the largest uptake according to size and intensity. Maximal SUV was calculated using the formula: $\mathrm{SUV}=\mathrm{C}_{\mathrm{dc}} /\left(\mathrm{d}_{\mathrm{i}} / \mathrm{w}\right)$, where $\mathrm{C}_{\mathrm{dc}}$ is the decay-corrected tracer tissue concentration (in becquerels per gram), $d_{i}$ is the injected dose (in becquerels), and $w$ is the patient's body weight (in grams) $(12,13)$.

Cell lines and cell culture. The cancer cell lines OVTOKO and OVISE were obtained both from JCRB Cell Bank (Osaka, Japan), and JHOC5 was obtained from RIKEN Cell Bank (Tsukuba, Japan) (14). These cell lines all derived from ovarian CCC and verified in writing as being ovarian in origin. They were cultured in RPMI-1640 medium or Dulbecco's modified Eagle's medium (DMEM) (both from Wako Pure Chemical Industries, Ltd., Osaka, Japan) supplemented with $10 \%$ fetal bovine serum (FBS; Invitrogen Life Technologies, Carlsbad, CA, USA), $100 \mathrm{U} / \mathrm{ml}$ penicillin, $100 \mu \mathrm{g} / \mathrm{ml}$ streptomycin and subcultured by $0.25 \%$ trypsin/EDTA (all from Wako Pure Chemical Industries, Ltd.) detachment. All of the cells were grown in a humidified atmosphere at $37^{\circ} \mathrm{C}$ and $5 \% \mathrm{CO}_{2}$.

Suspension (spheroid-forming) culture. Dissociated single cells $\left(2 \times 10^{5}\right.$ cells $\left./ \mathrm{ml}\right)$ were seeded into ultra-low attachment plates (Corning Inc., Corning, NY, USA) and were cultured for 2 days. For collecting spheroids, the medium was centrifuged at $100 \mathrm{x} g$ for $2 \mathrm{~min}$, and the supernatants were carefully aspirated.

Western blotting. The same amounts of protein from whole cell lysates were subjected to SDS-polyacrylamide gel electrophoresis (Bio-Rad Laboratories, Inc., Hercules, CA, USA) and electrotransferred onto polyvinylidene difluoride membrane (Millipore, Billerica, MA, USA). The membranes were blocked with $5 \%(\mathrm{w} / \mathrm{v})$ skim milk in TBS-Tween-20 for $1 \mathrm{~h}$ at room temperature. Then, the blots were probed with primary antibodies at 1:500 dilutions overnight at $4{ }^{\circ} \mathrm{C}$, followed by incubation with appropriate secondary antibodies conjugated to horseradish peroxidase (GE Healthcare) for $1 \mathrm{~h}$ at room temperature. The secondary antibodies were detected using Immobilon Western Chemiluminescent HRP Substrate (Millipore) according to the manufacturer's instructions.

Antibodies. Anti-CD44v6, anti-ASCT2 and anti-GLUT1 antibodies were purchased all from Abcam (Cambridge, MA, 


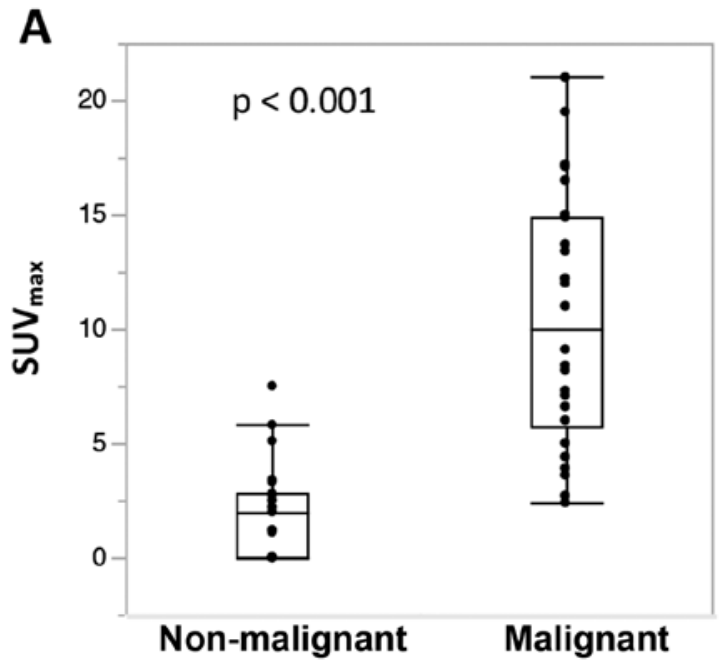

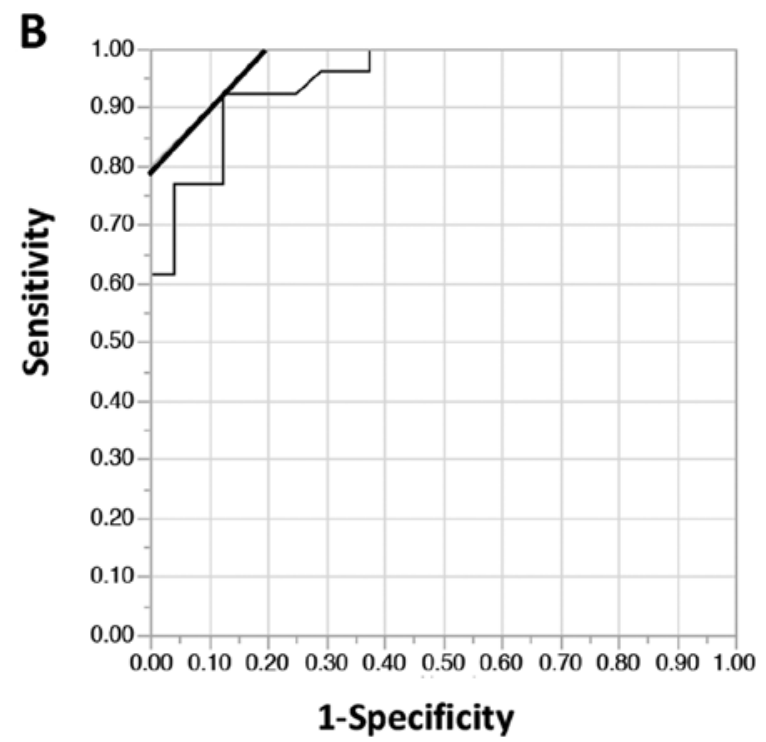

Figure 1. Diagnostic value of SUV $\mathrm{max}_{\text {ax }}$ and cut-off point. (A) Difference of SUV $\mathrm{max}_{\text {ax }}$ between non-malignant cases and malignant cases. Malignant cases had a significantly higher $\mathrm{SUV}_{\max }(\mathrm{p}<0.001)$ than non-malignant ones. (B) ROC curves for determining the cut-off value for predicting malignant tumors. The cut-off value for predicting malignant tumors was determined to be 3.6 with a sensitivity/specificity of $0.92 / 0.88$. SUV $\mathrm{V}_{\text {max }}$, maximum standardized uptake value; ROC, receiver operating characteristic.

USA) (cat. nos. 78960, 84903 and 40084, respectively). Anti-aldehyde dehydrogenase 1 (ALDH1) antibody was purchased from BD Biosciences (Franklin Lakes, NJ, USA) (cat. no. 611195). Anti-LAT1, anti-p-AMP-activated protein kinase (p-AMPK) and anti-AMPK antibodies were purchased all from Cell Signaling Technology, Inc. (Danvers, MA, USA) (cat. nos. 5347, 2535 and 2532, respectively). Anti- $\alpha$-tublin antibody was purchased from Millipore (cat. no. CP06).

Statistical analysis. The Wilcoxon singed-rank test was used to compare the medians. Logistic regression analysis was used to assess the association between max and malignant/non-malignant tumors. ROC curves were used to assess the criterion value. P-values $<0.05$ were considered statistically significant. JMP/SAS Institute was used for statistical analysis.

\section{Results}

Patient characteristics. To define the cut-off value, a total of 50 patients with ovarian tumors who underwent FDG-PET/CT before primary surgery were enrolled. A total of 24 cases were pathologically confirmed as non-malignant (benign and borderline) tumors, and 26 cases were confirmed as malignant tumors. The median age of the patients with non-malignant tumors was 43.5 years (range, 24-79 years). The median age of the patients with malignant tumors was 48 years (range, 22-79 years). The malignant tumors included serous carcinoma (19\%), mucinous carcinoma $(8 \%)$, endometrioid carcinoma (27\%) and CCC (35\%) (Table I).

Diagnostic value of $S U V_{\max }$ and cut-off point. The median $\mathrm{SUV}_{\max }$ for non-malignant tumors was 2.0 (range, 0-7.5). The median $\mathrm{SUV}_{\max }$ for malignant tumors was 10.1 (range, 2.4-21.0). Malignant cases had a significantly higher $\mathrm{SUV}_{\max }$ as shown in Fig. $1 \mathrm{~A}(\mathrm{p}<0.001)$. The cut-off value for predicting
Table I. Clinical FIGO stage and histologic type of malignant cases.

\begin{tabular}{lccccc}
$\begin{array}{l}\text { Clinical } \\
\text { stages }\end{array}$ & Clear & Endometrioid & Mucinous & Serous & Other \\
\hline I $(n=17)$ & 6 & 7 & 1 & 1 & 2 \\
II $(n=1)$ & 0 & 0 & 0 & 1 & 0 \\
III $(n=5)$ & 2 & 0 & 0 & 2 & 1 \\
IV $(n=3)$ & 1 & 0 & 1 & 1 & 0
\end{tabular}

Other, 1 seromucinous, 1 adenosarcoma, 1 serous + endometrioid (mixed).

malignant tumors was 3.6 with a sensitivity/specificity of 0.92/0.88, which was determined by ROC curves (Fig. 1B).

Cancers with a low $S U V_{\max }$. In the literature, cut-off values for predicting ovarian malignant tumors ranged from 2.5 to $3.7(4,8,10)$. These differences could come from the histological differences and the numbers of borderline tumors included. It is also known that SUV varies according to the facility's protocol for conducting FDG/PET (15). Although there was no definition for 'low $\mathrm{SUV}_{\text {max }}$ ', we considered 'below 4.0' as 'low $\mathrm{SUV}_{\max }$ '. We identified ovarian cancer patients with a low $\mathrm{SUV}_{\max }$ as described in the Materials and methods section. Eight patients were found to have a low $\mathrm{SUV}_{\max }$. The clinicopathological characteristics of these patients are shown in Table II. We found that most of the cases were CCC. Patients with ovarian CCC were often identified in the early stages (FIGO stage I/II). However, as shown in Fig. 2A, the population of cases in the early stages could not necessarily explain the low $\mathrm{SUV}_{\max }$ of CCC, suggesting that a specific biology of CCC should cause the low $\mathrm{SUV}_{\max }$. Furthermore, among patients with $\mathrm{CCC}$, the characteristic of 

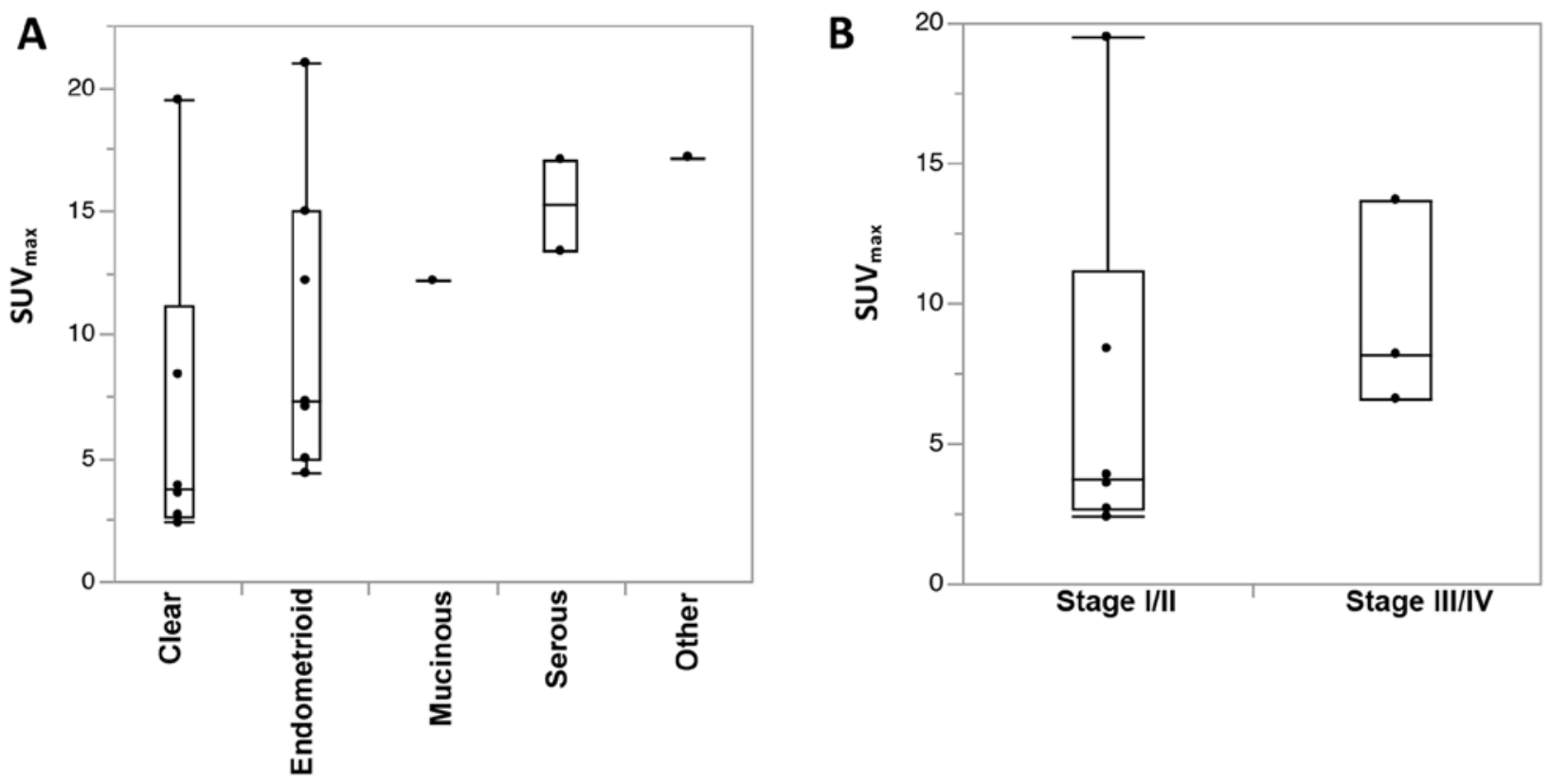

Figure 2. $\mathrm{SUV}_{\max }$ of different histological malignancies and stages. (A) $\mathrm{SUV}_{\max }$ of different histological types of ovarian carcinoma in early stages (FIGO stage I/II). Patients with ovarian CCC had a lower $\mathrm{SUV}_{\max }$ compared with other histological types in early stages. (B) $\mathrm{SUV}_{\max }$ of ovarian CCC between FIGO stage I/II and stage III/IV. A low SUV max $_{\text {in }}$ patients with CCC was especially characteristic of early-stage cases. Note that patients of CCC were picked up from those in Table I. SUV $\mathrm{Smax}_{\text {, }}$ maximum standardized uptake value; CCC, clear cell carcinoma.

Table II. Characteristics of patients with a low $\mathrm{SUV}_{\max }$.

\begin{tabular}{lcrlc}
\hline Patients & Age (years) & Stage & Histology & SUV $_{\text {max }}$ \\
\hline 1 & 70 & I & clear & 2.7 \\
2 & 43 & I & clear & 3.6 \\
3 & 43 & I & clear & 3.9 \\
4 & 64 & II & clear & 3.9 \\
5 & 51 & I & other & 3.3 \\
6 & 65 & I & clear & 3.1 \\
7 & 54 & III & other & 3.5 \\
8 & 36 & I & clear & 2.3 \\
\hline
\end{tabular}

Other, patient 5 , seromucinous, patient 7 , clear + endometrioid (mixed). The patients that were picked up here were different from those in Table I as described in Materials and methods. SUV $\mathrm{Sax}_{\max }$, maximum standardized uptake value.

a low $\mathrm{SUV}_{\max }$ is thought to apply in particular to early-stage cases (Fig. 2B).

Metabolic features of CCC in vitro. To investigate and gain insight into the metabolic features of $\mathrm{CCC}$, we then proceeded to conduct an in vitro experiment. We previously reported that OVTOKO, a CCC cell line, had different metabolic features between its cells with CSC-like properties and its non-CSCs (11). We also found that CSCs had significantly higher concentrations of glutamine than non-CSCs. Taken together, we hypothesized that the CSC-like properties should explain the low $\mathrm{SUV}_{\max }$ of CCC. We added the cancer cell lines of CCC, OVISE and JHOC5 and obtained cells with CSC-like properties by forming spheroid (described later, CSCs). Although a CSC marker for ovarian CCC has yet to be identified, we applied

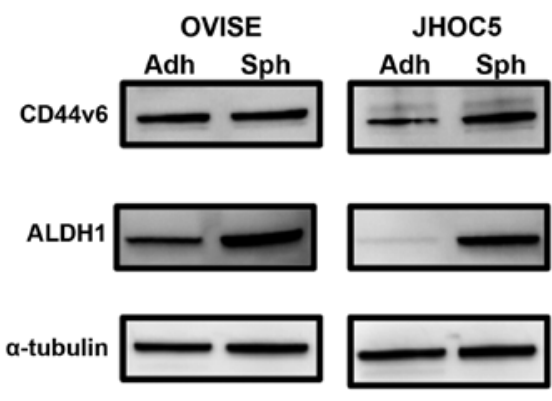

Figure 3. Expression of CSC markers in OVISE and JHOC5. The expression of CD44v6 was not increased in CSCs from OVISE but was increased CSCs from JHOC5 compared with non-CSCs. The expression of ALDH1 was increased in CSCs from both OVISE and JHOC5. CSC, cancer stem cell; ALDH1, aldehyde dehydrogenase 1; Adh, adherent cultured cells (later, non-CSC); Sph, spheroids (later, CSCs).

CD44v6 and ALDH1 as CSC markers (16,17). The expression of CD44v6 was not increased in CSCs from OVISE but was increased in CSCs from JHOC5 compared with respective adherent non-CSCs (Fig. 3). The expression of ALDH1 was increased in CSCs from both OVISE and JHOC5 (Fig. 3). Next, the expression levels of representative transporters that have significant roles in metabolism were investigated by western blotting. Whereas the expression level of ASCT2, a glutamine influx transporter, was nearly unchanged between non-CSCs and CSCs, the expression levels of LAT1, a glutamine efflux transporter, and GLUT1, a glucose influx transporter, were decreased in CSCs compared to non-CSCs, except for in JHOC5 (Fig. 4A). These changes indicated that there was metabolic reprograming between non-CSCs and CSCs. Indeed, the phosphorylation levels of AMPK, which is thought to be one of the metabolic sensors and switches (18), were reduced in CSCs compared to non-CSCs (Fig. 4B). 

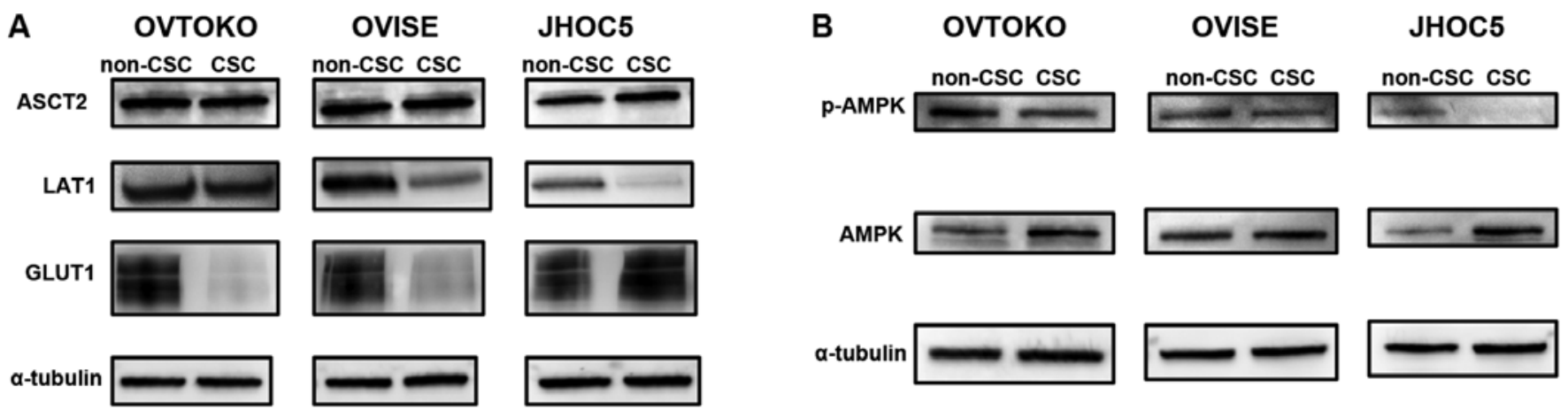

Figure 4. Expression level of transporters and metabolic sensors between non-CSCs and CSCs. (A) Expression level of transporters relating to glycolysis and glutaminolysis. Whereas the expression level of ASCT2, glutamine influx transporter, was nearly unchanged from non-CSCs to CSCs, the expression levels of LAT1, glutamine efflux transporter, and GLUT1, glucose influx transporter, were decreased in CSCs compared to non-CSCs, except for in JHOC5. (B) Phosphorylation level of AMPK. The phosphorylation levels of AMPK, which is thought to be one of the metabolic sensors and switches, were reduced in CSCs compared to non-CSCs. CSC, cancer stem cell; ASCT2, alanine, serine, cysteine-preferring transporter 2; LAT1, system L-type amino acid transporter 1; GLUT1, glucose transporter 1; AMPK, AMP-activated protein kinase.

\section{Discussion}

Here, we demonstrated that ovarian CCC had a low uptake of FDG in FDG-PET/CT, and this may reflect glutaminolysis of its CSC-like properties.

Cancers have two major pathways of metabolism. One is glycolysis, and the other is glutaminolysis $(1,9)$.

One technique in clinical practice that takes advantage of glycolysis is the FDG-PET/CT $(2,3)$. Glucose is transported into the cytoplasm via GLUTs, especially GLUT1. FDG is a glucose analog that is also transported into tissues, like glucose, but is not further metabolized, unlike glucose, resulting in its accumulation in high-glucose-using tissues, which is how FDG-PET/CT is used as a diagnostic aid to distinguish cancer cells from normal cells (5). One of the measurements that reflect the activation of glycolysis is the $\mathrm{SUV}_{\text {max }}$. Indeed, many studies have suggested that this tool not only has diagnostic value, but it also has prognosis predicting value (6-8).

As mentioned above, however, cancer cells do not always show activation of glycolysis (or high $\mathrm{SUV}_{\max }$ ) because they can also consume glutamine. Accordingly, we became interested in the characteristics of cancer cells with a low activation of glycolysis (low $\mathrm{SUV}_{\max }$ ).

In the first half of this study in which we identified cases with a low $\mathrm{SUV}_{\max }$ among patients with ovarian cancer, we showed that most of the cases were limited to patients with ovarian CCC (especially cases in early stages).

It is important to consider that a low uptake of $\mathrm{SUV}_{\max }$ does not always indicate a tumor biology of glycolysis but can indicate technical limitations of the FDG-PET/CT. For instance, ovarian mucinous carcinoma tends to have a low $\mathrm{SUV}_{\max }$ because of its low tumor cellularity and high amount of mucin (5). Hepatocellular adenocarcinoma expresses GLUT1 and uptakes glucose but has a low $\mathrm{SUV}_{\max }$ because it has glucose-6-phosphatase, which de-phosphorylates and effluxes FDG (19). Conditions such as high concentrations of serum glucose and inflammation make it difficult to detect the expected accumulation of FDG $(15,20)$. However, it is empirically known that biologically, ovarian CCC has a lower uptake of glucose compared with carcinoma of other histological types $(5,10)$. Indeed, one study demonstrated that ovarian CCC cell lines had a lower uptake of FDG than cell lines from other histological types in vitro (21).

In the latter half of this study, we performed an in vitro experiment to investigate the metabolic biology of CCC, which could explain its low $\mathrm{SUV}_{\max }$. In particular, we focused on the metabolic differences between CSCs and non-CSCs.

We obtained cells with CSC-like properties by forming spheroids as previously described (11). Then, we compared the expression levels of transporters between non-CSCs and CSCs, which correlate with cancer metabolism (western blotting). Whereas the expression level of ASCT2, a glutamine influx transporter, was nearly unchanged between non-CSCs and CSCs, the expression levels of system LAT1, a glutamine efflux transporter, and GLUT1, a glucose influx transporter, were decreased in CSCs compared to non-CSCs, except for in JHOC5. These patterns of changes in transporter expression could mean that glutamine metabolism had a prominent part in CSC metabolism. Although the expression of GLUT1 was increased in CSCs compared to non-CSCs in JHOC5, the concomitant decrease in LAT1 expression could still indicate that glutamine accumulation was important in the CSCs of JHOC5. Recent studies have demonstrated that the high expression levels of LAT1 and the high uptake of glucose were correlated with poor prognosis in ovarian (clear cell) carcinoma $(4,22-24)$. These facts may reflect the status in which CSCs differentiate and propagate into non-CSC, indicated by our in vitro data.

Some studies referred to the low $\mathrm{SUV}_{\max }$ of CCC, and they often attributed it to lower expression levels of GLUT1 or lower cell proliferation compared with other histological types of cancers $(5,10)$. In this study, we propose a new possible explanation, by comparing the metabolic features not between CCC and other histological types of cancers but between CSCs and non-CSCs in ovarian CCC. It is that the low $\mathrm{SUV}_{\max }$ of CCC may represent its reliance on glutaminolysis compared to glycolysis, and that it may be CSCs that account for the activation of glutaminolysis.

The limitation of our study is that we only obtained indirect evidence that glutaminolysis was significant in the CSCs of CCC. Although we confirmed that the phosphorylation level of AMPK, which is one of the metabolic sensors and has 
been recently indicated to be related to glycolysis $(18,25)$, was reduced in CSCs compared to non-CSCs, this remains indirect evidence of metabolic reprograming between non-CSCs and CSCs. Ideally, performing glutamine-based PET in patients with ovarian cancer should provide direct biological information about glutamine metabolism. Indeed, fluoroglutamine (FGln)-PET was recently introduced as a diagnostic tool for gliomas (26). We expect that this technology would not only be a tool for diagnosis, but combined with FDG-PET, it could also help investigate tumor metabolic behavior. Studies from this perspective are expected in the future.

In conclusion, we suggest that ovarian $\mathrm{CCC}$ has a low uptake of FDG-PET, and this may reflect glutaminolysis of its CSC-like properties.

\section{References}

1. Dang CV: Rethinking the Warburg effect with Myc micromanaging glutamine metabolism. Cancer Res 70: 859-862, 2010.

2. Sarker A, Im HJ, Cheon GJ, Chung HH, Kang KW, Chung JK, Kim EE and Lee DS: Prognostic implications of the $S_{U} V_{\text {max }}$ of primary tumors and metastatic lymph node measured by ${ }^{18} \mathrm{~F}$-FDG PET in patients with uterine cervical cancer: a meta-analysis. Clin Nucl Med 41: 34-40, 2016.

3. Yamamoto M, Tsujikawa T, Fujita Y, Chino Y, Kurokawa T, Kiyono Y, Okazawa $\mathrm{H}$ and Yoshida Y: Metabolic tumor burden predicts prognosis of ovarian cancer patients who receive platinum-based adjuvant chemotherapy. Cancer Sci 107: 478-485, 2016.

4. Kim C, Chung HH, Oh SW, Kang KW, Chung JK and Lee DS Differential diagnosis of borderline ovarian tumors from stage I malignant ovarian tumors using FDG PET/CT. Nucl Med Mol Imaging 47: 81-88, 2013.

5. Konishi H, Takehara K, Kojima A, Okame S, Yamamoto Y, Shiroyama Y, Yokoyama T, Nogawa T and Sugawara Y: Maximum standardized uptake value of fluorodeoxyglucose positron emission tomography/computed tomography is a prognostic factor in ovarian clear cell adenocarcinoma. Int J Gynecol Cancer 24: 1190-1194, 2014.

6. Chung HH, Kwon HW, Kang KW, Park NH, Song YS, Chung JK, Kang SB and Kim JW: Prognostic value of preoperative metabolic tumor volume and total lesion glycolysis in patients with epithelial ovarian cancer. Ann Surg Oncol 19: 1966-1972, 2012.

7. Risum S, Loft A, Engelholm SA, Høgdall E, Berthelsen AK, Nedergaard L, Lundvall L and Høgdall C: Positron emission tomography/computed tomography predictors of overall survival in stage IIIC/IV ovarian cancer. Int J Gynecol Cancer 22: $1163-1169,2012$.

8. Yamamoto Y, Oguri H, Yamada R, Maeda N, Kohsaki S and Fukaya T: Preoperative evaluation of pelvic masses with combined ${ }^{18} \mathrm{~F}$-fluorodeoxyglucose positron emission tomography and computed tomography. Int J Gynaecol Obstet 102: 124-127, 2008

9. Jin L, Alesi GN and Kang S: Glutaminolysis as a target for cancer therapy. Oncogene 35: 3619-3625, 2015.

10. Tanizaki Y, Kobayashi A, Shiro M, Ota N, Takano R, Mabuchi Y, Yagi S, Minami S, Terada M and Ino K: Diagnostic value of preoperative SUV $\mathrm{S}_{\max }$ on FDG-PET/CT for the detection of ovarian cancer. Int J Gynecol Cancer 24: 454-460, 2014.

11. Sato M, Kawana K, Adachi K, Fujimoto A, Yoshida M, Nakamura H, Nishida H, Inoue T, Taguchi A, Takahashi J, et al: Spheroid cancer stem cells display reprogrammed metabolism and obtain energy by actively running the tricarboxylic acid (TCA) cycle. Oncotarget 7: 33297-33305, 2016.
12. Kadoya T, Aogi K, Kiyoto S, Masumoto N, Sugawara Y and Okada M: Role of maximum standardized uptake value in fluorodeoxyglucose positron emission tomography/computed tomography predicts malignancy grade and prognosis of operable breast cancer: a multi-institute study. Breast Cancer Res Treat 141: 269-275, 2013

13. Park JC, Lee JH, Cheoi K, Chung H, Yun MJ, Lee H, Shin SK, Lee SK and Lee YC: Predictive value of pretreatment metabolic activity measured by fluorodeoxyglucose positron emission tomography in patients with metastatic advanced gastric cancer: the maximal SUV of the stomach is a prognostic factor. Eur J Nucl Med Mol Imaging 39: 1107-1116, 2012.

14. Kashiyama T, Oda K, Ikeda Y, Shiose Y, Hirota Y, Inaba K, Makii C, Kurikawa R, Miyasaka A, Koso T, et al: Antitumor activity and induction of TP53-dependent apoptosis toward ovarian clear cell adenocarcinoma by the dual PI3K/mTOR inhibitor DS-7423. PLoS One 9: e87220, 2014.

15. Keyes JW Jr: SUV: standard uptake or silly useless value? J Nucl Med 36: 1836-1839, 1995.

16. Mizuno T, Suzuki N, Makino H, Furui T, Morii E, Aoki H, Kunisada T, Yano M, Kuji S, Hirashima Y, et al: Cancer stem-like cells of ovarian clear cell carcinoma are enriched in the ALDH-high population associated with an accelerated scavenging system in reactive oxygen species. Gynecol Oncol 137: 299-305, 2015.

17. Tjhay F, Motohara T, Tayama S, Narantuya D, Fujimoto K, Guo J, Sakaguchi I, Honda R, Tashiro H and Katabuchi H: CD44 variant 6 is correlated with peritoneal dissemination and poor prognosis in patients with advanced epithelial ovarian cancer. Cancer Sci 106: 1421-1428, 2015.

18. Kishton RJ, Barnes CE, Nichols AG, Cohen S, Gerriets VA, Siska PJ, Macintyre AN, Goraksha-Hicks P, de Cubas AA, Liu T, et al: AMPK is essential to balance glycolysis and mitochondrial metabolism to control T-ALL cell stress and survival. Cell Metab 23: 649-662, 2016.

19. Horsager J, Bak-Fredslund K, Larsen LP, Villadsen GE Bogsrud TV and Sørensen M: Optimal 2-[(18)F] fluoro-2-deoxy-D-galactose PET/CT protocol for detection of hepatocellular carcinoma. EJNMMI Res 6: 56, 2016.

20. Shida M, Murakami M, Tsukada H, Ishiguro Y, Kikuchi K, Yamashita E, Kajiwara H, Yasuda M and Ide M: F-18 fluorodeoxyglucose uptake in leiomyomatous uterus. Int J Gynecol Cancer 17: 285-290, 2007.

21. Lutz AM, Ray P, Willmann JK, Drescher C and Gambhir SS: 2-Deoxy-2-[F-18]fluoro-D-glucose accumulation in ovarian carcinoma cell lines. Mol Imaging Biol 9: 260-266, 2007.

22. Fan X, Ross DD, Arakawa H, Ganapathy V, Tamai I and Nakanishi T: Impact of system L amino acid transporter 1 (LAT1) on proliferation of human ovarian cancer cells: a possible target for combination therapy with anti-proliferative aminopeptidase inhibitors. Biochem Pharmacol 80: 811-818, 2010.

23. Kaira K, Nakamura K, Hirakawa T, Imai H, Tominaga $H$, Oriuchi N, Nagamori S, Kanai Y, Tsukamoto N, Oyama T, et al: Prognostic significance of L-type amino acid transporter 1 (LAT1) expression in patients with ovarian tumors. Am J Transl Res 7: 1161-1171, 2015.

24. Lamkin DM, Spitz DR, Shahzad MM, Zimmerman B, Lenihan DJ, Degeest K, Lubaroff DM, Shinn EH, Sood AK and Lutgendorf SK: Glucose as a prognostic factor in ovarian carcinoma. Cancer 115: 1021-1027, 2009.

25. Saito Y, Chapple RH, Lin A, Kitano A and Nakada D: AMPK protects leukemia-initiating cells in myeloid leukemias from metabolic stress in the bone marrow. Cell Stem Cell 17: 585-596, 2015.

26. Venneti S, Dunphy MP, Zhang H, Pitter KL, Zanzonico P, Campos C, Carlin SD, La Rocca G, Lyashchenko S, Ploessl K, et al: Glutamine-based PET imaging facilitates enhanced metabolic evaluation of gliomas in vivo. Sci Transl Med 7: 274ra17, 2015. 